Originalien

Med Klin Intensivmed Notfmed 2021 · 116:586-594 https://doi.org/10.1007/s00063-020-00712-0 Eingegangen: 31 . Januar 2020

Überarbeitet: 8. Juni 2020

Angenommen: 4. Juli 2020

Online publiziert: 7. August 2020

(c) Der/die Autor(en) 2020

Redaktion

M. Buerke, Siegen

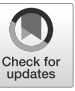

J. Berendt ${ }^{1} \cdot$ C. Ostgathe ${ }^{1} \cdot$ S. T. Simon ${ }^{2} \cdot$ M. Tewes ${ }^{3} \cdot$ D. Schlieper ${ }^{4}$. M. Schallenburger ${ }^{4} \cdot$ S. Meier ${ }^{5} \cdot$ S. Gahr ${ }^{1} \cdot$ J. Schwartz ${ }^{4} \cdot$ M. Neukirchen ${ }^{4,5}$

'Palliativmedizinische Abteilung, Universitätsklinikum Erlangen, Comprehensive Cancer Center EMNErlangen, Friedrich-Alexander-Universität Erlangen-Nürnberg (FAU), Erlangen, Deutschland

${ }^{2}$ Zentrum für Palliativmedizin, Centrum für Integrierte Onkologie Aachen, Bonn, Köln, Düsseldorf, Uniklinik Köln, Köln, Deutschland

${ }^{3}$ Westdeutsches Tumorzentrum, Innere Klinik (Tumorforschung), Universitätsklinikum Essen, Essen, Deutschland

${ }^{4}$ Interdisziplinäres Zentrum für Palliativmedizin, Universitätsklinikum, Heinrich-Heine-Universität Düsseldorf, Düsseldorf, Deutschland

${ }^{5}$ Klinik für Anästhesiologie, Universitätsklinikum, Heinrich-Heine-Universität Düsseldorf, Düsseldorf, Deutschland

\title{
Zusammenarbeit von Intensivmedizin und Palliativmedizin
}

\section{Eine Bestandsaufnahme an den deutschen onkologischen Spitzenzentren}

ner palliativmedizinischen stationären Behandlung profitieren. Hierzu ist eine frühzeitige Integration palliativer Prinzipien in die Intensivmedizin möglich [19, 22]. Die S3-Leitlinie „Palliativmedizin für Patienten mit einer nicht heilbaren Krebserkrankung“ empfiehlt generell eine frühzeitige Integration („early integration“) bei Tumorpatienten. Patienten sowie deren Vorsorgebevollmächtigte, Betreuer und Angehörige sollen bereits bei Diagnosestellung einer lebenslimitierenden Erkrankung über die Möglichkeiten der Palliativversorgung informiert werden. Außerdem soll ihnen während des stationären Aufenthalts der Kontakt mit einem Palliativdienst angeboten werden [16]. Auch bei Patienten mit lebenslimitierenden nichtonkologischen Grunderkrankungen ist ein früh einsetzendes Behandlungskonzept sinnvoll, damit die Palliativmedizin die krankheitsspezifische Therapie bzw. Begleitung mit besonderem Fokus auf die verbleibende Lebenszeit in einem nichtklinischen Setting und zum Teil mit spezialisierter palliativmedizinischer Ausrichtung aufgrund bestmöglicher
Lebensqualität unterstützen kann [3, 19, $20]$.

Diese Empfehlung gilt ebenso für Patienten, die intensivmedizinisch behandelt werden. In der Literatur sind vielfältige Kriterien für eine Bitte um Mitbehandlung seitens des intensivmedizinischen Teams zu finden [1]. Jedoch werden nicht alle Kriterien von den Intensivärzten akzeptiert und angewendet [2]. Die konsequente Einbindung der Krankenpflege kann helfen, Patienten mit Bedarf an Palliativmedizin zu identifizieren, ohne dass starre Kriterien vorgegeben werden [11, 13].

In deutschen Krankenhäusern sind es überwiegend die Kliniken für Anästhesiologie [6] und Innere Medizin, die zu einem erheblichen Teil sowohl zur Intensivversorgung als auch zur Palliativversorgung beitragen. Obwohl bereits einige Krankenhäuser einen palliativmedizinischen Dienst etabliert haben, fehlt es häufig an weiteren Versorgungsstrukturen und konkreten Maßnahmen der Integration von Palliativmedizin. Hinzu kommt im klinischen Alltag eine heterogene Akzeptanz und Nutzung palliativmedizinsicher Angebote sowohl von 
Hier steht eine Anzeige.

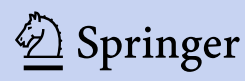


Tab. 1 Mitbehandlungen durch den Palliativdienst und Übernahmen von Patienten aus der intensivmedizinischen Versorgung an den einzelnen Comprehensive Cancer Centern (CCC)

\begin{tabular}{|l|l|l|l|}
\hline $\begin{array}{l}\text { CCC } \\
\text { Nr. }\end{array}$ & $\begin{array}{l}\text { Einbindung in Palliativ- } \\
\text { dienst (Anzahl der Patien- } \\
\text { ten) }\end{array}$ & $\begin{array}{l}\text { Übernahmen von ICU auf Pallia- } \\
\text { tivstation (Anzahl der Patienten) }\end{array}$ & $\begin{array}{l}\text { Palliativdienst } \\
\text { verfügbar }\end{array}$ \\
\hline 1 & 100 & 30 & Ja \\
\hline 2 & 6 & 6 & Ja \\
\hline 3 & 40 & 5 & Ja \\
\hline 4 & 52 & 22 & Ja \\
\hline 5 & 0 & Keine Angabe & Nein \\
\hline 6 & 0 & 4 & Nein \\
\hline 7 & 25 & 10 & Ja \\
\hline 8 & 0 & Keine Angabe & Nein \\
\hline 9 & 0 & 2 & Nein \\
\hline 10 & 5 & 20 & Ja \\
\hline 11 & 0 & 1 & Ja \\
\hline 12 & 25 & 20 & Ja \\
\hline 13 & 33 & 7 & Ja \\
\hline 14 & 60 & Keine Angabe & Ja \\
\hline 15 & 100 & 20 & Ja \\
\hline
\end{tabular}

Patienten als auch aufseiten der Primärbehandler.

Die Stiftung Deutsche Krebshilfe fördert in Deutschland onkologische Spitzenzentren (Comprehensive Cancer Center [CCC]), um damit wegweisende Strukturen für die Krebsversorgung zu schaffen. Dabei wird eine Behandlung nach einheitlichen, hohen Qualitätsstandards angestrebt. Eine Bestandserhebung unter den CCC ist daher in der Lage, erste Empfehlungen abzuleiten, wie Versorgungsstrukturen im Schnittstellenbereich zwischen Intensiv- und Palliativmedizin idealerweise aussehen sollen. Darüber hinaus kann eine solche Erhebung Hinweise auf einen Verbesserungsbedarf geben. Daher soll in dieser Arbeit die Integration der spezialisierten Palliativmedizin in die Intensivmedizin an den deutschen CCC untersucht werden. Die Fragestellungen lauten: Wie ist die Palliativmedizin aus Sicht der leitenden ärztlichen Vertreter der spezialisierten Palliativmedizin bislang in die Intensivmedizin ihrer jeweiligen Einrichtung integriert? Wie viele Patienten erhalten eine palliativmedizinische Mitbehandlung oder werden direkt von Intensivstationen auf Palliativstationen übernommen? Welche Trigger führen zu einer palliativmedizinischen Mitbehandlung?

\section{Studiendesign und Untersuchungsmethode}

Es handelt sich um ein deskriptives Studiendesign mit quantitativen und qualitativen Daten. Für die Vollerhebung wurde ein Fragebogen entwickelt, der neben der hier dargestellten Zusammenarbeit zwischen Intensivmedizin und Palliativmedizin auch die Implementierung einer spezialisierten ambulanten palliativmedizinischen Sprechstunde, ärztlichen Rotation und von Palliativdiensten erfragte $[4,9]$. Der Fragebogen wurde an alle ärztlichen Leitungen der spezialisierten Palliativmedizin in den 16 von der Stiftung Deutsche Krebshilfe geförderten deutschen CCC verschickt. Die Befragten erhielten den Fragebogen im Juli 2017 und konnten ihn bis August 2017 per Fax beantworten. Die Fragen bezogen sich auf das Jahr 2016 [4, 9]. Die Fragen zur Einbindung der spezialisierten Palliativmedizin in die jeweilige Intensivstation (ICU) bzw. Überwachungsstation beinhalteten offene und geschlossene Fragen. CCC erprobt und nach Rückmeldung angepasst. Es wurden Häufigkeitsverteilungen, Mittelwerte, Median und die jeweiligen Streumaße („standard deviation" [SD]) ermittelt. Die Auswertungen der vorgegebenen Antwortoptionen und Der Fragebogen wurde zuvor in drei die Erfassung der Freitextangaben erfolgten in SPSS (IBM, Armonk, NY, USA). Die Freitextangaben wurden anschließend von zwei wissenschaftlichen Mitarbeitern unabhängig voneinander analysiert und kategorisiert. Dabei wurde die zusammenfassende Inhaltsanalyse nach Mayring angewendet, um induktiv Kategorien zu bilden, die miteinander abgeglichen und konsentiert wurden [21, 25]. Für die induktive Vorgehensweise spricht, dass bisher die Gründe für die Einbindung der spezialisierten Palliativmedizin in die intensivmedizinische Versorgung (Trigger) in der Literatur uneinheitlich definiert sind [1].

\section{Ergebnisse}

Von 16 kontaktierten CCC nahmen 15 (94\%) teil. In den 15 CCC bestanden 6 palliativmedizinische Einheiten seit über 10 Jahren und 7 palliativmedizinische Einheiten seit 5 bis 10 Jahren. In einem CCC lag das Gründungsjahr der spezialisierten Palliativmedizin 3 bis 4 Jahre zurück, während ein CCC noch keine palliativmedizinische Abteilung hatte. Elf der 15 Zentren (73\%) boten einen palliativmedizinischen Dienst an.

Der Palliativdienst wurde im Median bei 33 Intensivpatienten pro Jahr (Spanne: 0-100) eingebunden. Für die direkten Übernahmen von Patienten der Intensivstation auf die Palliativstation ergab sich im CCC-Netzwerk ein Median von 9 Übernahmen pro Jahr (Spanne: 1-30). Die berichteten Zahlen sind in • Tab. 1 aufgeführt.

In 2 der 15 CCC (13\%) wurden bisher regelmäßige intensivmedizinisch-palliativmedizinische Visiten durchgeführt. Ein drittes CCC gab an, intensivmedizinisch-palliativmedizinische Visiten nicht regelmäßig, aber bei Bedarf durchzuführen.

In ebenfalls 2 der 15 CCC (13\%) ist auf den Intensivstationen ein ScreeningTool für die Einbindung der spezialisierten Palliativmedizin etabliert.

Insgesamt benannten 11 Befragte Triggerfaktoren für ein Hinzuziehen des palliativmedizinischen Teams. Hierbei konnten 23 Triggerfaktoren erfasst werden, die in der täglichen Praxis genutzt werden. Diese lassen sich in drei Kate- 
Med Klin Intensivmed Notfmed 2021 • 116:586-594 https://doi.org/10.1007/s00063-020-00712-0

(c) Der/die Autor(en) 2020

\section{J. Berendt · C. Ostgathe · S. T. Simon · M. Tewes · D. Schlieper · M. Schallenburger · S. Meier · S. Gahr · J. Schwartz · M. Neukirchen Zusammenarbeit von Intensivmedizin und Palliativmedizin. Eine Bestandsaufnahme an den deutschen onkologischen Spitzenzentren}

\section{Zusammenfassung}

Hintergrund. Die interdisziplinäre Zusammenarbeit zwischen Intensivmedizin und Palliativmedizin kann die Versorgungsqualität verbessern. Das Ausmaß dieser Zusammenarbeit ist aber bisher kaum untersucht.

Ziel der Arbeit. Es sollten die angebotenen und in Anspruch genommenen palliativmedizinischen Unterstützungsangebote auf den Intensivstationen deutscher onkologischer Spitzenzentren erfasst werden.

Material und Methoden. Durchgeführt wurde eine quantitativ-qualitative, deskriptive Umfrage an den 16 von der Stiftung Deutsche Krebshilfe geförderten Zentren. Die im quantitativen Teil erfragten Häufigkeiten werden als Mittelwert und Median mit den jeweiligen Streumaßen dargestellt, während die im qualitativen Teil erhobenen Triggerfaktoren mit einer Inhaltsanalyse nach Mayring ausgewertet wurden.

Ergebnisse. Von Juli bis August 2017 konnten Angaben aus 15 von 16 onkologischen Spitzenzentren (94\%) erfasst werden. Im Jahr 2016 wurden im Median 33 Intensivpatienten (Min. 0, Max. 100) palliativmedizinisch vorgestellt und 9 Patienten (Min. 1, Max. 30) auf eine Palliativstation verlegt. Regelmäßige intensivmedizinisch-palliativmedizinische Visiten sowie ein Screening-Tool zur Einbindung der spezialisierten Palliativmedizin sind an zwei onkologischen Spitzenzentren implementiert. Anhand von 23 genannten Triggern, die auf der Intensivstation eine palliativmedizinische Mitbehandlung ausgelöst haben, lassen sich nach qualitativer Analyse die drei Kategorien
"Entscheidung und Einstellung des Teams", "Zustand des Patienten" und "Wunsch von Patienten und Angehörigen" ableiten. Diskussion. Trotz eines verfügbaren Angebots werden palliativmedizinische Ressourcen in den intensivmedizinischen Abteilungen der onkologischen Spitzenzentren immer noch selten genutzt. In die tägliche Routine integrierte Angebote wie Screening-Tools oder gemeinsame Visiten könnten die Ausnutzung der angebotenen palliativmedizinischen Ressourcen erhöhen und die Versorgungsqualität verbessern.

Schlüsselwörter

Triggerfaktoren · Intensivpatient · Therapiezielfindung · Palliativdienst · Interdisziplinäre Zusammenarbeit

\section{Cooperation between intensive care and palliative care. The status quo in German Comprehensive Cancer Centers}

\section{Abstract}

Background. For intensive care patients with limited life expectancy the integration of palliative care in intensive care may be beneficial. However, little is known about the extent of this interdisciplinary collaboration. Objectives. The support given by palliative medicine in German oncological centers and used by the intensive care units should be recorded.

Material and methods. A descriptive survey was conducted in all of the 16 Comprehensive Cancer Centers (CCC) funded by German Cancer Aid. The questionnaires were sent to the head of department of the CCCs' specialized palliative care teams. Data were collected for the year 2016. Quantitative data were analysed to establish frequencies, given as mean and median. A qualitative section asked for trigger factors, i.e., patient characteristics triggering a palliative care consultation. Evaluation was inductively carried out by content analysis according to Mayring.

Results. Data from 15 of the 16 CCCs (94\%) were obtained between July and August 2017. In 2016, the median of intensive care patients with palliative care consultations was 33 (minimum 0, maximum 100). The median of nine patients were transferred from an intensive care unit to a palliative care unit (minimum 1, maximum 30). Multidisciplinary ward rounds by both intensive and palliative care staff were available in two CCCs on a regular basis. Two CCCs implemented screening tools to integrate specialized palliative care into intensive care. From 23 responses concerning triggers, three categories were established, i.e., "team's decision and attitude", "patient's condition" and "desires of patients and relatives". Conclusions. Palliative care is available in German CCCs. However, the degree of integration of specialized palliative care into intensive care units is low. Screening tools are available to identify patients with complex needs and to trigger a palliative care consultation. These tools, as well as joint ward rounds of intensive and palliative care staff, can improve the quality of patient centred care.

Keywords

Trigger factors - Intensive care patient . Therapeutic goal setting · Palliative care consultation service $\cdot$ Interdisciplinary collaboration gorien zusammenfassen: „Entscheidung und Einstellung des Teams“, „Zustand des Patienten“ und „Wunsch von Patienten und Angehörigen“ (• Tab. 2).

\section{Diskussion}

Die Zusammenarbeit von Palliativmedizin und Intensivmedizin in den deutschen onkologischen Spitzenzentren ist sehr unterschiedlich ausgestaltet. Ursächlich hierfür sind zunächst strukturelle Unterschiede des palliativmedizinischen Angebots. Lücken in der Verfügbarkeit spezialisierter palliativmedizinischer Angebote können Auswirkungen auf die Arbeit der Teams von Intensivstationen haben, wie natio- nal und international festgehalten wird $[18,24]$. Während nahezu alle Standorte Palliativstationen vorhielten (und vorhalten), boten in 2016 nur ca. $70 \%$ der Zentren einen palliativmedizinischen Dienst an. Diese beratenden Dienste als ein Modell der Palliativversorgung für die Intensivpflege [18] stellen ein elementares Bindeglied zwischen statio- 
Tab. 2 Triggerfaktoren zur Einbindung des Palliativdiensts

\begin{tabular}{|c|c|c|c|}
\hline $\begin{array}{l}\text { Häufigkeit der } \\
\text { Nennungen }\end{array}$ & Genannter Trigger (Freitext) & $\begin{array}{l}\text { Generalisierung (Häufigkeit } \\
\text { der Nennungen) }\end{array}$ & $\begin{array}{l}\text { Kategorie (Häufig- } \\
\text { keit der Nennungen) }\end{array}$ \\
\hline 3 & Therapiezielklärung, -begrenzung, -änderung & \multirow[t]{2}{*}{ Therapiezieländerung (4) } & \multirow{11}{*}{$\begin{array}{l}\text { Entscheidung und } \\
\text { Einstellung des } \\
\text { Teams (13) }\end{array}$} \\
\hline 1 & $\begin{array}{l}\text { Therapieeskalation erfolgt durch die Kollegen direkt in Absprache mit } \\
\text { den Angehörigen }\end{array}$ & & \\
\hline 1 & $\begin{array}{l}\text { Persönlich-individuelle Entscheidung, Einstellung der Ärzte (mitarbei- } \\
\text { tergetriggert) }\end{array}$ & \multirow[t]{2}{*}{$\begin{array}{l}\text { Entscheidung und Einstellung } \\
\text { der Mitarbeiter (2) }\end{array}$} & \\
\hline 1 & Einstellung der Ärzte & & \\
\hline 1 & Bedarf für palliativmedizinischen Sozialdienst & \multirow[t]{2}{*}{ Unklare Versorgung (2) } & \\
\hline 1 & Unklare Weiterversorgung & & \\
\hline 1 & $\begin{array}{l}\text { Vorgespräch mit Patienten/Angehörigen zur geplanten palliativmedi- } \\
\text { zinischen Versorgung }\end{array}$ & $\begin{array}{l}\text { Vorgespräch zur palliativmedi- } \\
\text { zinischen Versorgung (1) }\end{array}$ & \\
\hline 1 & $\begin{array}{l}\left.\text { Visite des Oberarzthintergrunddiensts der Klinik [... }{ }^{a}\right] \text { (u. a. Oberärzte } \\
\text { mit palliativmedizinischer Weiterbildung) }\end{array}$ & \multirow[t]{2}{*}{$\begin{array}{l}\text { Palliativmedizinische } \\
\text { Zusatzweiterbildung (2) }\end{array}$} & \\
\hline 1 & $\begin{array}{l}\text { Sehr komplexe Verhältnisse }\left[\ldots{ }^{a}\right] \text {, in einigen Stationen }{ }^{\mathrm{a}} \text { sind qualifizier- } \\
\text { te Palliativmediziner mit Zusatzweiterbildung verantwortlich tätig }\end{array}$ & & \\
\hline 1 & Ggf. persönliche Bekanntheit & \multirow[t]{2}{*}{ Persönlicher Kontakt (2) } & \\
\hline 1 & Persönliche Ansprache & & \\
\hline 2 & Symptome (symptomatisch und/oder psychologisch) & Symptome (2) & \multirow{5}{*}{$\begin{array}{l}\text { Zustand des } \\
\text { Patienten (6) }\end{array}$} \\
\hline 1 & Nicht heilbare Grunderkrankung & $\begin{array}{l}\text { Nicht heilbare Grunderkran- } \\
\text { kung (1) }\end{array}$ & \\
\hline 1 & Sterbephase & Sterbephase (1) & \\
\hline 1 & Belastetes Familiensystem & \multirow[t]{2}{*}{ Belastete Situation (2) } & \\
\hline 1 & Schwierige ethische Situation & & \\
\hline 2 & Angehörigenwunsch & \multirow{3}{*}{$\begin{array}{l}\text { Übernahmewunsch von } \\
\text { Patienten und } \\
\text { Angehörigen (4) }\end{array}$} & \multirow{3}{*}{$\begin{array}{l}\text { Wunsch von Patienten } \\
\text { und Angehörigen (4) }\end{array}$} \\
\hline 1 & Patientenwunsch & & \\
\hline 1 & Übernahmewunsch Palliativstation & & \\
\hline
\end{tabular}

när palliativ- und intensivmedizinischer Versorgung dar und sollten daher ausgebaut werden. Sowohl die S3-Leitlinie „Palliativmedizin für Patienten mit einer nicht heilbaren Krebserkrankung“ [16] als auch die Best-Practice-Empfehlung der Stiftung Deutsche Krebshilfe [27] sieht in jedem CCC sowohl eine Palliativstation als auch einen palliativmedizinischen Dienst vor. Durch das Zusatzentgelt ZE 2018-133 sind hier erst in den letzten Jahren neue potenzielle Finanzierungsmöglichkeiten geschaffen worden, sodass, wenn das Zusatzentgelt kalkuliert sein wird, mit einer besseren Versorgung zu rechnen ist. Nach erfolgreicher Etablierung der strukturellen Voraussetzungen für eine spezialisierte palliativmedizinische Behandlung an den CCC stellen diese eine Vorbildfunktion für weitere Versorger dar. Obwohl in vielen CCC Strukturen zur palliativmedizinischen Mitbehandlung zur Verfügung stehen [5] und die Inan- spruchnahme des Palliativdiensts von anderen Abteilungen regelhaft erfolgt [7, 10], ist die Anzahl der Anfragen der Intensivmedizin an die Palliativmedizin in den meisten CCC bisher noch verhältnismäßig gering, zum Beispiel unter Einbezug der Ergebnisse einer österreichischen Studie [19]. Die vorliegende Arbeit zeigt auf, dass es nicht grundsätzlich an einer Lücke in der generellen Verfügbarkeit eines Palliativdiensts liegt und demnach auch Modelle zur Integration von Palliativmedizin in die Intensivmedizin $[18,19,28]$ in die Anwendung kommen sollten. Regelmäßige (z.B. zweiwöchentliche) intensivmedizinisch-palliativmedizinische Visiten, die sich zu einem unmittelbaren Booster palliativmedizinischer Aktivität auf einer Intensivstation entwickeln können [19], sowie standardisierte ScreeningTools werden auch an den Zentren mit existierenden Palliativdiensten nur unregelmäßig angeboten.
Im Alltag werden Intensivmediziner im Schnitt einmal pro Woche mit Therapiezieländerungen konfrontiert, in deren Rahmen eine zunächst kurativ intendierte Behandlung in eine palliative Behandlung überführt wird [14]. $35 \%$ der Ärzte fühlen sich bei dieser Entscheidung unsicher [14]. Insbesondere deshalb muss sichergestellt sein, dass zum einen genügend Intensivmediziner mit palliativmedizinischem Basiswissen zur Verfügung stehen und dass zum anderen im Falle hoher Symptomlast die zur Verfügung stehenden Strukturen der spezialisierten Palliativmedizin bekannt sind [29, 30]. Außerdem sollte ein standardisiertes Vorgehen zur Anforderung des Palliativdiensts etabliert sein [15]. Bisher konnte diesbezüglich jedoch noch unzureichendes Wissen festgestellt werden $[15,29,30]$. Die ärztliche und pflegerische palliativmedizinische Aus-, Fort- und Weiterbildung in den CCC bedarf der Weiterentwicklung 
Hier steht eine Anzeige.

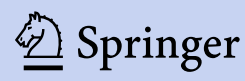


[14]. Kommunikative Fähigkeiten zur Übermittlung schlechter Nachrichten am Lebensende sind verbesserungsfähig [31]. Auch wenn sich viele Patienten ihres unmittelbar bevorstehenden Lebensendes bewusst sind, hat nur ein geringerer Anteil der Patienten diesen Punkt mit einem Arzt besprochen [12]. Jedoch werden Therapiezieländerungen zunehmend standardisiert dokumentiert, z.B. in Anlehnung an den Dokumentationsbogen der Sektion Ethik der Deutschen Interdisziplinären Vereinigung für Intensiv- und Notfallmedizin $[13,17,23,26]$. Patienten und deren Vorsorgebevollmächtigte befürworten zu Beginn eines protrahierten postoperativen Verlaufs häufig Maximaltherapie, was sich im Krankheitsverlauf jedoch ändern kann [12]. Eine derartige Meinungsänderung ist oft mit dem Wunsch nach einer Therapiezieländerung verknüpft und betrifft insbesondere Patienten, die durch Langzeitintensivtherapie hoch belastet sind, die einen schlechten funktionellen Status aufweisen oder die nach langer Intensivbehandlung unter reduzierter Lebensqualität leiden. Ein Palliativdienst kann dann im Laufe der Behandlung für Patienten, Angehörige und die behandelnden Berufsgruppen ergänzend unterstützen. Mit dem Behandlungsfokus auf Symptomkontrolle und Lebensqualität kann der Patient bis zum Lebensende auf der Intensiv- oder Normalstation verbleiben. Ein gewisser Prozentsatz der Intensivpatienten bedarf nach palliativmedizinischer Mitbehandlung aber auch einer Übernahme auf die Palliativstation [8]. Der Anteil der von einem Palliativdienst mitbehandelten Patienten, die in der Folge auf die Palliativstation übernommen wurden, liegt in unserer Untersuchung bei $27 \%$. Dass nicht jeder mitbehandelte Patient, selbst nach Therapiezieländerung auf ein palliatives Therapieziel, auf die Palliativstation übernommen werden kann, ist unter anderem darauf zurückzuführen, dass Therapiezieländerungen auf Intensivstationen häufig mit der Einstellung von Organersatzverfahren einhergehen (z.B. Katecholamintherapie bei Herzinsuffizienz oder Sepsis, Beatmung bei respiratorischer Insuffizienz oder kontinuierliche Nierenersatztherapie bei akutem Nierenversagen). Zumeist treten diese Patienten dann sehr schnell in die Sterbephase ein, in der eine Verlegung, verbunden mit einem belastenden Umgebungswechsel, nicht mehr indiziert bzw. vertretbar ist. Außerdem führt nicht jede Therapiezieländerung zur Einstellung sämtlicher intensivmedizinischer Maßnahmen, sodass auch dann eine Verlegung in die spezialisierte Palliativmedizin nicht möglich ist [18].

Screening-Tools und die darin genannten standardisierten Triggerfaktoren könnten die Häufigkeit palliativmedizinischer Mitbehandlungen auch auf Intensivstationen erhöhen, doch unter Intensivmedizinern besteht nur eine geringe Akzeptanz bisher publizierter Faktoren [2]. Die im Rahmen der qualitativen Inhaltsanalyse erfassten Trigger decken sich nahezu vollständig mit denen in der bisher veröffentlichten Literatur [1]. Lediglich die Gruppe der Triggerfaktoren mit dem Schwerpunkt „Entscheidung und Einstellung des Teams" wird von den befragten leitenden Ärzten der Palliativmedizin als zusätzlicher Trigger genannt. Sowohl bei den befragten Palliativmedizinern als auch bei jungen Intensivmedizinern scheinen die beiden Aspekte „Wunsch von Patienten und Angehörigen “ und „Zustand des Patienten“ die akzeptiertesten Auslöser für eine palliativmedizinische Mitbehandlung zu sein [2]. Diesbezüglich wären weitere Studienergebnisse bezogen auf die Sinnhaftigkeit einzelner Triggerfaktoren für eine palliativmedizinische Mitbehandlung von Intensivpatienten wünschenswert, um dann evidenzbasiert Screening-Tools zu entwickeln. Regelmäßige intensivmedizinisch-palliativmedizinische Visiten könnten $\mathrm{zu}$ einer Optimierung (und damit Vereinheitlichung) der Behandlung beitragen. Im Rahmen dieser Visite werden in einem gemeinsamen Entscheidungsprozess von primärbehandelnden Intensivmedizinern und Palliativmedizinern Intensivpatienten identifiziert, die von einer multiprofessionellen palliativmedizinischen Mitbehandlung profitieren können. Solche gemeinsamen Visiten werden aktuell nur in 2 der CCC regelmäßig durchgeführt.
Die Ergebnisse zeigen, dass die Etablierung eines palliativmedizinischen Diensts in einem CCC (und damit allgemein in jedem größeren Krankenhaus) möglich ist. Das Vorhandensein eines palliativmedizinischen Diensts stellt eine wichtige Grundvoraussetzung für die fachübergreifende Zusammenarbeit zwischen der Intensiv- und Palliativmedizin dar. Screening-Tools und gemeinsame Visiten von Intensivmedizinern und Palliativmedizinern helfen dabei, mögliche Palliativpatienten auf Intensivstationen frühzeitig zu erkennen [19]. Diese hilfreichen Tools sind bisher allerdings auch in den CCC nur selten etabliert, sodass eine weitere Verbreitung zum Beispiel im Rahmen zukünftiger Zertifizierungen vorgeschlagen werden könnte.

\section{Limitationen}

Die Studie basiert auf einer Befragung einer hochselektionierten Kohorte. Dennoch liegt den Ergebnissen bei Antworten aus 15 von 16 geförderten CCC eine Vollerhebung zugrunde. Befragt wurden die leitenden Ärzte der spezialisierten Palliativmedizin. Intensivmediziner könnten den Bedarf anders einschätzen und vor allem die Trigger für eine Anfrage oder für einen Übernahmewunsch anders werten. Gründe für eine Nichtmitbehandlung durch ein spezialisiertes palliativmedizinisches Team werden nicht erfasst. Unbeleuchtet bleibt dadurch, inwiefern ein Bewusstsein des palliativmedizinischen Angebots und ein Abwägen seitens des intensivmedizinischen Teams, bevor Konsilanfragen an den Palliativdienst ausgeschlossen oder gestellt werden, tragend sind. Hierzu empfiehlt sich eine eigenständige qualitative und/ oder quantitative Erhebung. Eine weitere Limitation der Ergebnisse besteht darin, dass Schätzwerte für die Angaben zur Übernahmerate bzw. zu den getätigten Mitbehandlungen berücksichtigt wurden, da die Freitextangaben teilweise mit „ca." oder „ “ deklariert waren oder eine Spanne angegeben wurde. Im Falle einer Spanne wurde bei der Auswertung der Maximalwert hinzugezogen. Die Problematik angegebener Spannwerte ist auf die Papierversion des Fragebogens zurückzuführen. Ein Online-Frage- 
bogen mit vordefinierten Feldern hätte eine Angabe von Spannwerten verhindert. Bei der Papierversion des Fragebogens hätte sich der schriftliche Hinweis empfohlen, dass lediglich nur eine Zahl anzugeben ist. Die Gesamtzahl der intensivmedizinisch behandelten Patienten bleibt unberücksichtigt, sodass die erhobenen Zahlen nicht ins Verhältnis gesetzt werden konnten. Zuweisungszahlen anderer Abteilungen wurden nicht erhoben und verglichen. Bei der Abfrage wurde nicht zwischen onkologischen und nichtonkologischen Patienten unterschieden, da die Zahlen zu klein ausgefallen wären. Die Bestandserhebung gibt schlaglichtartig den Bestand des Jahres 2016 wieder. Eine erneute, erweiterte Umfrage ist wünschenswert, um eine zeitliche Entwicklung ableiten zu können.

\section{Fazit für die Praxis}

- Der Palliativdienst stellt für die Versorgung von Intensivpatienten neben der Palliativstation ein Kernelement der spezialisierten palliativmedizinischen Versorgung dar.

- Die Gründe für eine palliativmedizinische Mitbehandlung von Intensivpatienten lassen sich einordnen in die Kategorien „Entscheidung und Einstellung des Teams", "Zustand des Patienten" und "Wunsch von Patienten und Angehörigen".

- Regelmäßige intensivmedizinischpalliativmedizinische Visiten und standardisierte Screening-Tools können helfen, Intensivpatienten mit palliativmedizinischem Bedarf zu identifizieren.

- Ein nicht unerheblicher Anteil der Intensivpatienten bedarf nach palliativmedizinischer Mitbehandlung einer Übernahme auf die Palliativstation.

\section{Korrespondenzadresse}

\section{Dr. J. Schwartz}

Interdisziplinäres Zentrum für Palliativmedizin Universitätsklinikum, Heinrich-Heine-

Universität Düsseldorf

Moorenstraße 5, 40225 Düsseldorf,

Deutschland

jacqueline.schwartz@med.uni-duesseldorf.de

Funding. Open Access funding provided by Projekt DEAL.

\section{Einhaltung ethischer Richtlinien}

Interessenkonflikt. Alle Autoren (J. Berendt, C. Ostgathe, S.T. Simon, M. Tewes, D. Schlieper, M. Schallenburger, S. Meier, S. Gahr, J. Schwartz und M. Neukirchen) sind oder waren Angestellte eines der beteiligten CCC.

Für diesen Beitrag wurden von den Autoren keine Studien an Menschen oder Tieren durchgeführt. Für die aufgeführten Studien gelten die jeweils dort angegebenen ethischen Richtlinien.

Open Access. Dieser Artikel wird unter der Creative Commons Namensnennung 4.0 International Lizenz veröffentlicht, welche die Nutzung, Vervielfältigung, Bearbeitung, Verbreitung und Wiedergabe in jeglichem Medium und Format erlaubt, sofern Sie den/die ursprünglichen Autor(en) und die Quelle ordnungsgemäßnennen, einen Link zur Creative Commons Lizenz beifügen und angeben, ob Änderungen vorgenommen wurden.

Die in diesem Artikel enthaltenen Bilder und sonstiges Drittmaterial unterliegen ebenfalls der genannten Creative Commons Lizenz, sofern sich aus der Abbildungslegende nichts anderes ergibt. Sofern das betreffende Material nicht unter der genannten Creative Commons Lizenz steht und die betreffende Handlung nicht nach gesetzlichen Vorschriften erlaubt ist, ist für die oben aufgeführten Weiterverwendungen des Materials die Einwilligung des jeweiligen Rechteinhabers einzuholen.

Weitere Details zur Lizenz entnehmen Sie bitte der Lizenzinformation auf http://creativecommons.org/ licenses/by/4.0/deed.de.

\section{Literatur}

1. Adler K, Schlieper D, Kindgen-Milles D, Meier S, SchwartzJ, van CasterP, SchaeferMS, Neukirchen M (2017) Integration der Palliativmedizin in die Intensivmedizin: Systematische Übersichtsarbeit. Anaesthesist 66:660-666

2. Adler K, Schlieper D, Kindgen-Milles D, Meier S, Schallenburger $M$, Sellmann T, Schwager $H$, Schwartz J, Neukirchen M (2018) Will your patient benefit from palliative care? A multicenter exploratory survey about the acceptance of trigger factors for palliative care consultations among ICU physicians. Intensive Care Med 45:125-127

3. Alt-Epping B, Geyer A, Nauck F (2008) Palliativmedizinische Konzepte bei nicht-onkologischen Grunderkrankungen. Dtsch Med Wochenschr 133:1745-1749
4. Berendt J, Thomas M, Neukirchen M, Schwartz J, Hense J, Tewes M (2018) Integration von Palliativmedizin in onkologische Spitzenzentren Deutschlands - ambulante Sprechstunden und Rotationsprogramme der spezialisierten Palliativmedizin. Dtsch Med Wochenschr 143:e139-e145

5. Berendt J, Oechsle K, Thomas M, van Oorschot B, Schmitz A, Radbruch L, Simon S, Gärtner J, ThußPatience P, Schuler U, Hense J, Gog C, Viehrig M, Mayer-Steinacker R, Stachura P, Stiel S, Ostgathe C (2016) Integration der Palliativmedizin in die von der Deutschen Krebshilfe e. V. geförderten onkologischen Spitzenzentren. Dtsch Med Wochenschr 141:e16-e23

6. Erlenwein J, Geyer A, Schlink J, Petzke F, Nauck F, Alt-Epping B (2014) Characteristics of a palliative care consultation service with a focus on pain in a German university hospital. BMC Palliat Care 13(13):45

7. Erlenwein J, PetzkeF, StamerU, MeißnerW, NauckF, Pogatzki-Zahn E, Koppert W, Maier C (2017) Rolle der Anästhesiologie in der schmerzmedizinischen und palliativmedizinischen Versorgung in deutschen Krankenhäusern: Befragung von Chefärzten der Anästhesiologie zu Versorgungsstrukturen. Anaesthesist 66:579-588

8. Gaertner J, Frechen S, Sladek M, Ostgathe C, Voltz R (2012) Palliative care consultation service and palliative care unit: Why do we need both? The Oncol 17:428-435

9. Gahr S, Berendt J, Lödel S, Ostgathe C, Simon ST, Tewes M, Zader K, Schwartz J, Neukirchen M (2019) Palliativdienste in den deutschen onkologischen Spitzenzentren. Dtsch Med Wochenschr 144:e153-e159

10. Homsi J, Walsh D, Nelson KA, LeGrand SB, Davis M, Khawam E, Nouneh C (2002) The impact of a palliative medicine consultation service in medical oncology. Support Care Cancer 10:337-342

11. Hopprich A, Reinholz U, Gerlach C, Weber M (2018) How to identify the inpatient's palliative care needs in a hemato-oncological ward: Ask the nurses! JPalliat Med 21:4-5

12. Hwang IC, Keam B, Yun YH, Ahn HY, Kim Y-A (2015) Quality of life changes and intensive care preferences in terminal cancer patients. Palliat Support Care 13:1309-1316

13. Janssens U, BurchardiH, Duttge G, ErchingerR, GretenkortP, Mohr M, NauckF, RothärmelS, Salomon F, Schmucker P, Simon A, Stopfkuchen H, Valentin A, Weiler N, Neitzke G (2013) Therapiezieländerung und Therapiebegrenzung in der Intensivmedizin. Positionspapier der Sektion Ethik der Deutschen Interdisziplinären Vereinigung für Intensiv- und Notfallmedizin. Med Klin Intensivmed Notfmed 108:47-52

14. Jox RJ, Krebs M, Fegg M, Reiter-Theil S, Frey L, Eisenmenger W, Borasio GD (2010) Limiting lifesustaining treatment in German intensive care units: a multiprofessional survey. J Crit Care 25:413-419

15. Kamel G, Paniagua M, Uppalapati A (2015) Palliative care in the intensive care unit: Are residents well trained to provide optimal care to critically ill patients? Am J Hosp Palliat Med 32:758-762

16. Leitlinienprogramm Onkologie der AWMF, Deutsche Krebsgesellschaft und Deutsche Krebshilfe (Hrsg.) (Hrsg) (2019) Erweiterte S3-Leitlinie Palliativmedizin für Patienten mit einer nicht heilbaren Krebserkrankung. Langversion 2.0. 27.08.2019. https://www.awmf.org/leitlinien/detail/ll/1280010L.html.Zugegriffen:23.Dez. 2019 
17. Leitlinienprogramm Onkologie der AWMF, Deutsche Krebsgesellschaft und Deutsche Krebshilfe (Hrsg). Therapiezielfindung und Kriterien der Entscheidungsfindung. In Zitat Nr. 2

18. Lemm H, Hoeger-Schäfer J, Buerke M (2018) Palliativbehandlung. Herausforderungen auf der Intensivstation. Med Klin Intensivmed Notfmed 113:249-255

19. Lenz K, Hofmann-Bichler B, Pihringer J, Firlinger F, Pickl A, Clodi M (2017) Palliative Care auf der Intensivstation. Deskriptive Analyse der Ergebnisse eines Mischmodells am einerinternistischen Intensivstation über einen Zeitraum von 12 Monaten. Med Klin Intensivmed Notfmed 112:724-730

20. Ma J, Chi S, Buettner B, Pollard K, Muir M, Kolekar C, Al-Hammadi N, Chen L, Kollef M, Dans M (2019) Early palliative care consultation in the medical ICU: a cluster randomized crossover trial. Crit Care Med 47:1707-1715

21. Mayring P, Gläser-Zikuda M (2008) Die Praxis der qualitativen Inhaltsanalyse, 2. Aufl. Beltz, Weinheim Basel

22. Müller-Busch HC (2013) Palliativmedizin und Sterben aufder Intensivstation-kein Widerspruch. DIVI 1:22-27

23. Neitzke G, Böll B, Burchardi H, Dannenberg K, DuttgeG, ErchingerR, GretenkortP,Hartog C, KnochelK, Liebig M, Michalsen A, Michels G, Mohr M, Nauck F, Radke P, Salomon F, Stopfkuchen H, Janssens U (2017) Dokumentation der Therapiebegrenzung: Empfehlung der Sektion Ethik der Deutschen Interdisziplinären Vereinigung für Intensiv- und Notfallmedizin (DIVI) unter Mitarbeit der Sektion Ethik der Deutschen Gesellschaft für Internistische Intensivmedizin und Notfallmedizin (DGIIN). Med Klin Intensivmed Notfmed 112:527-530

24. Nelson J, Angus DC, Weissfeld LA, Puntillo KA Danis M, Deal D, Levy MM, Cook DJ (2006) End-oflife care for the critically ill: a national intensive care unit survey. Crit Care Med 34:2547-2553

25. Ramsenthaler C (2013) Was ist "Qualitative Inhaltsanalyse?". In: Schnell M, Schulz C, Kolbe H, Dunger $C$ (Hrsg) Der Patient am Lebensende. Springer, Wiesbaden, S23-42

26. Riessen R, Bantlin C, Wiesing U, Haap M (2013) Therapiezieländerungen auf einer internistischen Intensivstation: Einfluss von Willensäußerungen der Patienten auf Therapieentscheidungen. Med Klin Intensivmed Notfmed 108:412-418

27. Stiftung Deutsche Krebshilfe (2017) Ausschreibung zum Förderschwerpunkt Onkologische Spitzenzentren (7. Ausschreibungsrunde). https://www.krebshilfe.de/fileadmin/ Downloads/PDFs/Foerderung/CCCs_7th_call/ Ausschreibung_Leitfaden_7._Call_08_Sep_ 2017.pdf.Zugegriffen:23. Dez. 2019

28. Trzecak S (2015) Der Palliativpatient als Notfallpatient. Ein Modell zur Therapieentscheidung bei lebensbedrohlichen Situationen dargestellt anhand von 4 Kasuistiken. Med Klin Intensivmed Notfmed 110:278-286

29. Ward AM, Agar M, Koczwara B (2009) Collaborating or co-existing: a survey of attitudes of medical oncologists toward specialist palliative care. Palliat Med 23:698-707

30. Wentlandt K, Krzyzanowska MK, Swami N, Rodin GM, Le LW, Zimmermann C (2012) Referral practices of oncologists to specialized palliative care. JClin Oncol 30:4380-4386

31. Visser M, Deliens L, Houttekier D (2014) Physicianrelated barriers to communication and patientand family-centred decision-making towards the end of life in intensive care: a systematic review. Crit Care 18:604

\section{Broschüre informiert (laien-)verständlich über Herzoperation}

Kurz, prägnant, verständlich: Die neue Informationsbroschüre „Auf einen Blick Herzoperation“ der Deutschen Gesellschaft für Thorax,- Herz- und Gefäßchirurgie (DGTHG) in Zusammenarbeit mit der Deutschen Herzstiftung richtet sich an Patientinnen und Patienten, bei denen zur Behandlung ihrer Herzerkrankung eine Herzoperation notwendig ist oder war. „Eine Herzoperation ist ein einschneidendes Ereignis, welches Fragen aufwirft und Verunsicherungen hervorrufen kann. Daher ist die umfassende Aufklärung ein wichtiges Instrument, um etwaige Ängste zu nehmen, den Behandlungsablauf und das operative Verfahren zu erklären, und Antworten auf häufige Fragen sowie verständliche Informationen in diesem Zusammenhang zu geben", erklärt Prof. Dr. Andreas Böning, Herzchirurg und Präsident der DGTHG. Auf elf Seiten der neuen Broschüre werden operative Zugangswege dargestellt, körperliche Reaktionen auf eine Herzoperation aufgezeigt sowie Wundheilung, Rehabilitation und Genesung nachvollziehbar beschrieben. „Die individuelle Vorgehensweise besprechen Patientinnen und Patienten im Detail immer mit ihren betreuenden Ärztinnen und Ärzten in der herzchirurgischen Fachabteilung. Wir haben uns in der Broschüre auf Allgemeingültiges konzentriert", so Prof. Böning.

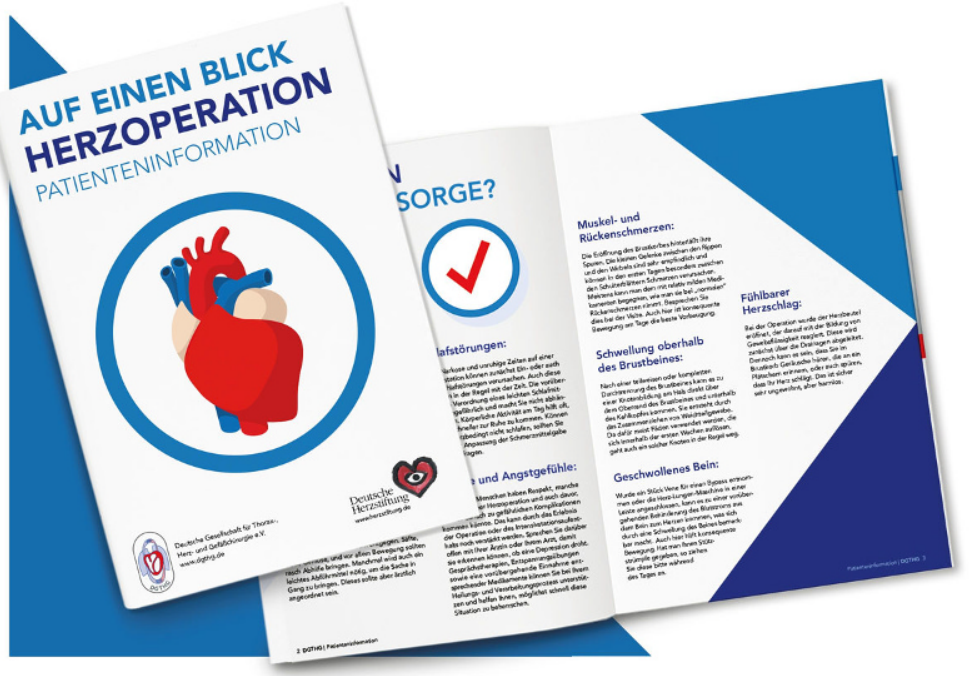

Patientinnen und Patienten, Interessierte und Krankenhäuser/Arztpraxen können die Broschüre kostenfrei als Print oder im digitalen Format (PDF) bei der Deutschen Herzstiftung anfordern.

Digital zum Download und Print-Produkt-Bestellung unter: https://www.herzstiftung.de/bestellung

Weitere Informationen unter www.dgthg.de und www.herzstiftung.de

Quelle:

Regina Iglauer-Sander, Pressereferentin,

Deutsche Gesellschaft für Thorax-, Herz- und Gefäßchirurgie e.V. 